\title{
Quel sujet écoutons-nous? De la structure subjective à la clinique du bien-dire
}

\author{
Paula Calado
}

\section{Résumé}

L'efficacité du travail psychanalytique et le fonctionnement d'un dispositif clinique guidé par la psychanalyse trouvent leur fondement dans une écoute qui oriente le traitement. L'émergence de la psychanalyse implique une décentralisation. La division entre Moi et Ça est claire dans les travaux de Freud, en particulier avec le concept de Spaltung, division, scission. Lacan, dans sa lecture de l'ouvrage freudien, propose une vaste construction théorique qui s'occupe de conceptualiser le sujet pour la psychanalyse. Le sujet comme le reste d'une division, comme la scansion, marqué par le langage se traduit par $\mathrm{S}$ barré (par le signifiant). La notion de $S$ barré est utilisée pendant des années par Lacan, jusqu'à un certain moment de son élaboration théorique, lorsque la notion de parlêtre entre en scène, pour rendre compte d'un sujet qui a un corps. La dimension corporelle n'était pas présente dans le premier enseignement lacanien et le parlêtre exprime le sujet le plus éloigné de la notion de signifiant et plus proche de la lettre de l'inconscient. La question qui se pose est de savoir si le changement conceptuel de $\mathrm{S}$ barré au parlêtre entraîne un changement dans l'écoute clinique et dans l'acte du psychanalyste. De plus, nous soutenons que l'étude théorique et la compréhension des deux moments de Lacan en ce qui concerne le sujet nous aident à penser une clinique plus éthique et à favoriser un désir perçu à la lettre.

\section{Mots-clés :}

Psychanalyse ; Sujet; S barré ; Parlêtre. 


\title{
What subject do we listen to? \\ From the subjective structure to the well-meaning clinic
}

\begin{abstract}
The effectiveness of the psychoanalytic work and the functioning of a clinical device guided by psychoanalysis are based on a listening process that guides the treatment. The emergence of psychoanalysis implies decentralization. The division between Me and It is clear in Freud's work, especially with the concept of Spaltung, division, split. Lacan, in his reading of the Freudian work, proposes a vast theoretical construction that deals with conceptualizing the subject for psychoanalysis. The subject, like the remainder of a division, like scansion, marked by language, is translated as barred S (by the signifier). The notion of barred $S$ is used for years by Lacan, until a certain moment of his theoretical elaboration, when the notion of parlêtre enters the scene, to account for a subject who has a body. The corporal dimension was not present in the first Lacanian teaching and the parletre expresses the subject furthest removed from the notion of signifier and closer to the letter of the unconscious. The question that arises is whether the conceptual change of barred $S$ to the parlêtre brings about a change in the clinical listening and the act of the psychoanalyst. In addition, we argue that the theoretical study and understanding of Lacan's two moments in the subject helps us to think of a more ethical clinic and to foster a desire perceived of the letter.
\end{abstract}

\section{Keywords:}

Psychoanalysis; Subject; Barred S; Parlêtre.

\section{Que sujeito escutamos? Da estrutura subjetiva à clínica do bem-dizer}

\begin{abstract}
Resumo
A eficiência do trabalho psicanalítico e o funcionamento de um dispositivo clínico orientado pela psicanálise encontram suas bases em uma escuta que dirige o tratamento. O surgimento da psicanálise implica uma descentralização. A divisão entre Eu e Isso fica clara na obra de Freud, sobretudo com o conceito de Spaltung, divisão, cisão. Lacan, em sua leitura da obra freudiana, propõe uma vasta construção teórica, que se dispõe a conceitualizar o sujeito para a psicanálise. $\mathrm{O}$ sujeito como resto de uma divisão, como escansão, marcado pela linguagem, traduz-se em S barrado (pelo significante). A noção de S barrado é utilizada durante
\end{abstract}


anos a fio por Lacan, até determinado momento de sua elaboração teórica, quando o conceito de parlêtre entra em cena, para dar conta de um sujeito que há um corpo. A dimensão de corpo não estivera presente no primeiro ensino lacaniano, e o parlêtre expressa o sujeito mais distante da noção de significante e mais próximo da letra do inconsciente. A questão que se apresenta é se a mudança conceitual de $S$ barrado a parlêtre propõe uma mudança na escuta clínica e na atuação do psicanalista. Mais além, defendemos que o estudo teórico e a compreensão dos dois momentos de Lacan no que diz respeito ao sujeito ajudam-nos a pensar uma clínica mais ética e a favor de um desejo que se apreende pela letra.

\title{
Palavras-chave:
}

Psicanálise; Sujeito; S barrado; Parlêtre.

\section{¿Que sujeto escuchamos? \\ De la estructura subjetiva a la clinica del bien-decir}

\begin{abstract}
Resumen
La efectividad del trabajo psicoanalítico y el funcionamiento de un dispositivo clínico guiado por el psicoanálisis se basan en un proceso de escucha que guía el tratamiento. La aparición del psicoanálisis supone una descentralización. La división entre Yo y Ello está clara en el trabajo de Freud, especialmente con el concepto de Spaltung, división. Lacan, en su lectura de la obra freudiana, propone una vasta construcción teórica que trata de conceptualizar el sujeto para el psicoanálisis. El sujeto como el resto de una división, como la exploración, marcada por el idioma, se traduce como $S$ barrado (por el significante). Lacan utiliza la noción de $S$ barrado a lo largo de años, hasta que en cierto momento de su elaboración teórica la noción de parlêtre surge, para explicar un sujeto que tiene un cuerpo. La dimensión corporal no estuvo presente en la primera enseñanza lacaniana y el parlêtre expresa el sujeto más alejado de la noción de significante y más cercano a la letra del inconsciente. La pregunta que surge es si el cambio conceptual de $\mathrm{S}$ a parlêtre provoca un cambio en la escucha clínica y el acto del psicoanalista. Además, argumentamos que el estudio teórico y la comprensión de los dos momentos de Lacan en el tema nos ayudan a pensar en una clínica más ética y favorable a un deseo que se apreende por la letra.
\end{abstract}

\section{Palabras clave:}

Psicoanálisis; Sujeto; S barrado; Parlêtre. 


\section{Introduction}

Le point le plus important d'un domaine d'études est l'objet de recherche, c'est-à-dire, le point central de la quête, le point qui pose une question au chercheur. Ce point principal en psychanalyse est l'inconscient, découvert par Freud et amplement étudié par Lacan. L'existence de l'inconscient présuppose l'existence d'un sujet de l'inconscient. Ce travail porte sur la question du sujet de l'inconscient d'après Lacan qui a comme point de départ une conception de $\$$, pensé à partir d'une structure signifiante, qui précède la notion de sujet parlêtre, associé à la lettre de l'inconscient. Un changement théorique s'opère dans le parcours de Lacan, vu qu'il part d'une conception de sujet fondé sur le symbolique vers une perspective nouvelle du sujet comme parlêtre, articulé au réel. Le but de ce travail est de mettre en remarque qu'un changement théorique en ce qui concerne le sujet de la psychanalyse implique un changement aussi de la clinique psychanalytique.

La question qui se pose est si le fait que Lacan a passé d'un moment à l'autre, du symbolique vers le réel, a contribué pour un changement au niveau de la clinique, de la direction d'une analyse. Une conception de sujet implique une conception de fantasme, un rapport avec l'objet $a$, et avec le désir. Ceci dit, j'envisage parler du chemin lacanien par rapport au sujet, dans le but d'éclairer la notion de fantasme et de direction de la cure. Pour faire ça, je suis allée chercher les arguments de Lacan dans les textes suivants : "La direction de la cure et les principes de son pouvoir », publié aux Écrits en 1958 (1966d) ; Le séminaire, livre V : Les formations de l'inconscient, de 1957-1958 (1998) ; Le séminaire, livre VI : Le désir et son interprétation, de 1958-1959 (2013) ; "Subversion du sujet et dialectique du désir dans l'inconscient freudien », de 1966b et « Radiophonie » (1970/2001d) ; texte court publié aussi sur les Autres écrits, qui date de 1970, c'est-à-dire, la fin de l'enseignement.

Penser le sujet en psychanalyse c'est penser au sujet de l'inconscient, celui des actes manqués, des rêves et des lapsus. Cette logique a permis à Freud de construire une doctrine qui considérait déjà que le sujet et le moi ne sont pas la même chose et que justement là où le moi n'est pas, le sujet surgit. À partir de cette notion, Lacan a fait une séparation entre sujet de l'énoncé et sujet de l'énonciation. C’est au Séminaire sur la lettre volée (Lacan, 1966a) que Lacan affirme que la grande découverte freudienne a été de situer le sujet de l'inconscient bien au-delà du moi. Cette découverte donne les bases pour la construction de toute une gamme de concepts psychanalytiques comme fantasme, répétition et trauma. Concepts qui s'appuient sur la logique d'un inconscient comme effet de l'Autre, et d'un sujet comme ce qui est représenté par un signifiant à un autre signifiant.

Enfin, ce travail propose une lecture vers l'importance d'une conception de sujet pour structurer la clinique et la transmission en psychanalyse. Qui est le sujet de l'inconscient lacanien et quelle est l'importance de l'étudier? Une perspective 
théorique sur le sujet serait-elle capable de produire des effets sur l'interprétation de l'analyste et pourrait-elle nous amener vers une clinique plus éthique?

\section{La barre du signifiant}

Ceux qui disent que les gens qu'on voit dans la rue peuvent être appelés sujets, certainement ne sont pas psychanalystes. Quelques psychologies ont pris le terme sujet pour désigner l'individuel, l'individu. Dans cette perspective, toute et n'importe quelle personne peut être appelée un sujet, vu que son individualité en tant que personne singulière fait qu'elle soit déjà un sujet. Pour la psychanalyse, le terme sujet est placé comme un concept, ce qui fait que, dans une lecture psychanalytique, un sujet n'est pas la même chose qu'une personne.

Quand on parle de sujet en psychanalyse on parle d'un être assujetti. Plus précisément, pour Lacan, le sujet n'a pas de substance, il n'est pas un corps vivant, mais quelque chose qui doit être convoqué pour se faire apparaître. Cette histoire commence avec Freud, quand il crée une doctrine de l'inconscient où la principale contribution est la découverte de la notion que nous n'avons pas le contrôle sur tous nos actes, que le moi n'est qu'une surface qui cache un sujet de désir, qui s'échappe et se montre dans les rêves, les lapsus et dans les symptômes.

Quand une personne va chez un psychanalyste, elle s'adresse à l'analyste comme un souffrant. Elle croit qu'il existe un savoir sur sa souffrance et adresse une question à l'analyste comme celui qu'il suppose savoir. Pour écouter le sujet du désir, de la répétition, et surtout, le sujet de la jouissance, l'analyste doit faire advenir le sujet de l'inconscient. Cette logique est ce qui détermine le discours de l'analyste comme un rapport entre $a$ et S barré, comme Lacan nous montre dans le Séminaire XVII (Lacan, 1969-1970/1991). Le fantasme, concept bien travaillé sur le Séminaire VI, démarque aussi un lien entre le sujet et l'objet $a$.

La découverte freudienne de l'inconscient opère une discontinuité, une excentricité par rapport au moi. Mettre en cause l'existence de quelque chose au-delà de la pensée consciente fait de la psychanalyse une des trois plus grandes déceptions de l'humanité. Darwin, Freud et Copernic ont retiré l'homme de la notion égocentriste. Lacan, à partir de Freud, a créé tout un parcours théorique et conceptuel, dans le but de structurer un sujet de la psychanalyse. Au début, une approche avec le monde de la linguistique pousse Lacan à interpréter le symbolique par le signifiant. Quand on se situe aux années 1958-1959, avec les cours du Séminaire VI (Lacan, 1958) et la publication du texte « Subversion du Sujet et dialectique du désir dans l'inconscient freudien » (Lacan, 1966b) on voit une définition de sujet qui se traduit à travers son rapport avec le signifiant, à savoir S barré.

Ce grand $S$ avec une barre est une création lacanienne démarque une phase de l'enseignement où le symbolique prenait une place fondamentale, et le $S$ barré 
démontre un rapport du sujet avec le langage. La première parution du concept de S barré dans l'enseignement de Lacan date de 1957-1958 dans le Séminaire V: Les formations de l'inconscient (1957-1958/1998). De ce texte on peut repérer une notion que la psychanalyse propose la convocation d'un sujet de l'inconscient, vu qu'il y a un écart entre ce qu'on dit et ce qu'on veut dire.

On localise aussi à la fin des années 1950, une position lacanienne qui insiste sur le fait que l'inconscient existe en rapport avec l'Autre. C'est l'introduction dans le monde du langage qui fait qu'une opération de division soit opéré par le signifiant, ce qui finit pour mettre la barre du côté du sujet, mais aussi de barrer l'Autre. Cette division est bien éclairée par Lacan sur le texte Direction de la cure (Lacan, 1966b), et fait référence au terme freudien de Spaltung. La refente ou division opérée par le langage résulte à un sujet articulé à un manque-à-être, vu que le produit de cette opération de division est l'objet $a$.

C'est en 1958 avec le Séminaire VI sur la logique du fantasme que Lacan fait l'approche du S barré avec l'objet $a$. Ce Séminaire très riche nous permet de comprendre la formule du fantasme : $\mathbb{S}<>a$, nous permettant de définir justement le rapport entre le sujet de l'inconscient et le reste de sa division comme le fantasme. Ceci dit, la naissance du sujet barré est articulée à la construction d'un fantasme. Ce fantasme qui se construit dans l'inscription du sujet dans le registre signifiant, mais qui doit se reconstruire à nouveau pendant une analyse, vu que le sujet ne s'y reconnaît. On y reviendra.

Le S barré est justement ce qui échappe au symbolique, ce qui fait discontinuité à la chaîne signifiante. Encore sur le texte « La direction de la cure » Lacan aborde les spécificités du sujet barré, comme celui qui est frappé par le signifiant, en faisant référence au texte freudien Un enfant est battu (Freud, 1919/1978). La notion que la frappe du signifiant opère la division du sujet oriente Lacan vers la construction de la logique du fantasme comme ce qui témoigne d'une jouissance propre au sujet qui est placée dans l'Autre.

La frappe signifiante qui met la barre et opère la division du sujet résulte en perte de jouissance, ce qui inscrit le rapport sexuel comme impossibilité. Le fantasme, alors, est structuré comme une tentative répétitive de retrouver la jouissance perdue, de chercher dans le champ de l'Autre la possibilité du rapport sexuel.

L'importance de la barre demeure sur le fait que l'Autre est aussi barré dans cette opération, ce qui lui donne inconsistance. Cette discontinuité dans l'Autre est ce qui permet le lien social. Quand l'opération de substitution du Désir de la Mère par la Métaphore Paternelle ne s'opère pas, la barre n'est pas mise dans l'Autre, ce qui lui donne un caractère de jouissance envahissante, comme on voit dans la psychose. La barre est définie comme une " marque invisible, qui aliène ce sujet dans l'identification première qui forme l'idéal de moi " (Lacan, 1966b, p. 808). L'objet $a$ est ce qui vient pour combler cette division et se structurer comme 
cause du désir. Le $\$$ est le sujet assujetti au langage, subordonné aux dits de l'Autre. Bien compris, le signifiant de l'Autre est ce qui produit le sujet barré, divisé, parlé avant même de naître.

On peut remarquer que dans ce premier moment de l'enseignement qui débute en 1957-1958, Lacan définit le sujet en même temps qu'il définit le signifiant. Je le cite «Un signifiant est ce qui représente un sujet à un autre signifiant » (Lacan, 1966b, p. 819). Cette définition, d’après Izcovich :

Elle désigne donc le sujet à partir de la chaîne signifiante et donne ainsi sa structure à partir de la façon dont la chaîne inconsciente exerce ses effets. La question que cela soulève est celle de la place du corps, qui reste vacante dans cette doctrine, et donc plus précisément de son articulation au concept de sujet. (Izcovich, 2008, p. 35)

Ceci dit, la première définition de sujet comme $S$ barré, comme celui qui fait scansion dans la chaîne signifiante, ne reconnaît pas le corps. Donner un corps à ce sujet inconsistant est une question qui se pose à Lacan aux années 1960 et un changement de perspective se met en scène quand il considère la jouissance par rapport au signifiant. À partir des années 1960, le réel gagne un autre statut est si avant le sujet était considéré comme celui qui tombe de la continuité de la chaîne signifiante, en 1972 il le définit comme « discontinuité dans le réel » (Lacan, 1966c, p. 310).

Considérer le réel et la jouissance dans ce deuxième moment nous amène à penser que Lacan a un changement de conception de sujet. Le parlêtre, sujet de son deuxième enseignement vient à la place du $\mathrm{S}$ barré. Ce concept vient réunir le sujet parlé par l'Autre et le sujet parlant, l'énoncé et l'énonciation. Le parlêtre introduit finalement la dimension de corps, comme vivant, qui manquait au S barré. Ce changement de perspective change le cours de l'enseignement, et au niveau de la clinique, le symptôme qui avant était considéré comme simple retour du refoulé, à ce moment-là peut être lu comme événement de corps, comme le point où le corps rejoint la jouissance. Selon Miller :

Le sujet qui, comme effet de signification, « est réponse du réel ». J’ai considéré cette phrase de Lacan comme surprenante. On a le sentiment que c'est vraiment trapu. Mais il faut s'apercevoir que le sujet, c'est-à-dire ces différentes élisions qui se promènent, c'est tout ce que nous avons comme réponse du réel. (...) Qu'est-ce que c'est la réponse du réel dans la psychanalyse, si cette réponse c'est le sujet et rien que ça ? Eh bien, si la réponse du réel c'est le sujet, on voit à quoi nous pouvons attendre au niveau du sujet. (Miller, 1983, p. 5) 
Bien compris, le réel prend une place importante dans le deuxième moment de l'enseignement de Lacan, ce qui modifie aussi les perspectives cliniques et l'acte de l'analyste. Un changement de perspective au niveau abstrait de la théorie provoque aussi un raffinement au niveau de l'écoute analytique. Comment la conception de sujet produit des effets sur la clinique?

\section{Désir <> fantasme}

La conception de sujet dans l'enseignement de Lacan ne doit pas être considérée comme une abstraction, vu qu'il y a une importance clinique en ce qui concerne la connaissance de ce sujet que l'analyse fait advenir. Cette notion met en question le processus analytique et la position de l'analyste. Quand on comprend, à partir de Lacan, que la spécificité de l'homme est sa forme de durer par le signifiant, on comprend qu'une écoute en psychanalyse va au-delà du dit. On doit emmener ce sujet au plus loin de sa singularité, mais comment faire ça?

Depuis la première page du Séminaire VI (Lacan, 1958), Lacan parle du travail de l'analyse. Les pages qui suivent présentent le pas-à-pas de la construction du graphe du désir pour mettre l'accent sur le fait que l'analyse travaille sur et à partir du désir. Le désir de savoir de l'analysant est nécessaire pour faire surgir une question, l'énigme du désir, « que me veut-il ?». De l'autre côté, il faut le désir opérateur, de l'analyste, celui décroché de son propre fantasme, qui opère pour faire surgir le trait de singularité chez le sujet, la différence absolue, comme Lacan dit à la fin du Séminaire XI (Lacan, 1963/1973).

D’après Lacan, le désir est le point énigmatique du sujet et l'analyse suit ses articulations dans le discours du sujet. C'est le travail du signifiant qui nous permet d'accéder quelque chose de l'ordre du désir, c'est-à-dire, les points de rupture de la chaîne signifiante nous révèlent la voie du désir. Je le cite :

Le désir est cet $x$ du sujet qui est pris dans le réseau signifiant, dans les mailles du signifiant, qui est soumis au filtrage, au criblage du signifiant. Nous avons pour but de le révéler, restituer, restaurer, dans son discours. Comment pouvons-nous le faire? que signifie que nous pouvons le faire? Selon la doctrine, la pratique, l'expérience, freudienne, je vous l'ai dit, la position du désir est d'être exclu, énigmatique. (Lacan, 1958, p. 171)

L'expérience analytique nous présente ainsi une stricte articulation entre l'être du sujet et le signifiant, vu qu'il est ce qui constitue le sujet en tant que tel et c'est la vacillation signifiante qui fait surgir un sujet du désir localisé dans la scansion, dans les points de rupture de la chaîne. "C'est fondamentalement le langage qui introduit la dimension de l'être pour le sujet, et en même temps la lui dérobe » 
(Lacan, 1958, p. 171). Ce point est capital dans la clinique, vu que la dimension d'existence d'un sujet est articulée à la marque du signifiant.

L'analyse consiste, donc, d'une reconstitution de ce qui a été une fois effacé mais reste ineffaçable chez le sujet. Une reconstruction du fantasme est nécessaire pour connaître l'adressage de ce discours, l'Autre de ce sujet, à qui il parle. L'analyste, décroché de son fantasme et vidé de ses identifications imaginaires est poussé par le désir de l'analyste, celui de l'ordre d'une opération, qui fait appel au sujet du désir, par la voie de l'interprétation. D’après Lacan, "Interpréter le désir, c'est restituer ceci auquel le sujet ne peut pas accéder à lui tout seul, à savoir l'affecte qui désigne son être, et qui se place au niveau du désir qui est le sien » (Lacan, 1958, p. 171).

Le rapport à l'être du sujet se déligne dans le champ du signifiant. Cette perspective fait que la psychanalyse ne se tient pas à l'idée du corps biologique ou du sujet du besoin, comme plusieurs courants psychologiques. Le sujet du besoin est celui de l'élan vitale, sur lequel s'appuie la médecine, par exemple. Quand on parle de sujet de l'inconscient on considère ce que le sujet veut au plus loin de son rapport à l'être, c'est-à-dire, ce que maintien son existence au niveau de son désir. Le sujet du besoin est articulé au moi, cette surface de rapport imaginaire au petit-autre qui est ensuite prise par le Grand Autre. Le moi, donc, institue un rapport à l'imaginaire et le désir, se fixe dans un rapport au fantasme. D’après les mots de Lacan :

Le sujet en tant qu'évanouissement, en tant qu'il s'évanouit dans un certain rapport à un objet électif — voilà le rapport que je vous désigne par le fantasme. Le fantasme a toujours cette structure. Il n'est pas simplement relation d'objet. Il est quelque chose qui coupe. C'est un certain évanouissement, une certaine syncope du sujet en présence d'un objet. Le fantasme satisfait à une certaine accommodation, fixation, du sujet par rapport à un objet ayant une valeur élective. (Lacan, 1958, p. 209)

La dimension du désir et la constitution du fantasme sont détaillées par Lacan à travers le graphe du désir, encore sur le Séminaire VI, il explique le passage d'un point à l'autre, ce qui nous permet de le lire comme ça :

Le $x$ qu'est le désir se situe quelque part sur la ligne qui revient du code inconscient, en sens inverse de la ligne intentionnelle qui est en face, soit le segment de retour $(\mathrm{S}<>\mathrm{D})$---> d. Le désir est là, flottant quelque part dans l'au-delà de l'Autre. Cependant, nous savons aussi que le désir est soumis à une certaine régulation. Celle-ci nous est ici représentée par sa 
hauteur de fixation, si l'on peut dire, au niveau d'un point déterminé de la ligne qui, revenant du message de l'inconscient, file en direction de s(A), le message de l'Autre sur le plan imaginaire. Cette ligne s'arrête à mi-course sur le $S<>a$, car c'est le fantasme qui règle la hauteur de fixation du désir, qui détermine sa situation. (Lacan, 1958, p. 337)

C'est-à-dire que le produit du désir qui souffre une régulation et s'articule au champ de l'Autre est le fantasme. Le fantasme dans son rôle de régulation du désir crée une réalité au sujet supportable à vivre. Cette fenêtre du réel détermine comment le sujet voit le monde, à partir d'un mythe où il construit son rapport au monde. La fin de l'analyse a été associée au travail de traverser le fantasme. Le début de l'analyse propose une construction du fantasme qui doit venir avant sa traversée, simultanée à la chute radicale de l'Autre.

\section{La clinique : du parlé au parlant}

L'importance de l'étude de la conception de sujet chez Lacan et ses différentes interprétations demeure sur le fait qu'à partir d'un éclairage de ce que c'est le sujet en question en psychanalyse, il est possible d'améliorer une écoute clinique et de, finalement cristalliser la pratique de l'analyste. Quand un sujet nous raconte son histoire il est encré par les signifiants de l'Autre, ce qui lui donne sa place au monde. Je me souviens d'une patiente à Sainte-Anne, qui, après avoir maigri de 32 kilos après la prise de sa retraite sort une formule de son père : "ceux qui ne travaillent pas, ne mangent pas". Et voilà le signifiant qui marque le corps et qui fait dit-mansion de l'existence du sujet.

L'acte analytique consiste justement en passer d'un moment à l'autre. Quand on parle d'éthique du désir et d'éthique du sujet on confirme l'idée que la psychanalyse est un contre-courant aux discours pragmatiques, adaptatifs ou médicales. Ce qui nous intéresse dans la parole d'un analysant est, effectivement, le point où il n'y est pas. L'intervalle des signifiants, le point de rupture d'un discours expose la dimension de sujet à laquelle on s'intéresse.

L'association libre, méthode freudienne, atteste depuis son origine que parmi les dits de l'Autre il y a un dire du sujet qui échappe à l'aliénation radical, un trait de singularité et d'inventivité dont l'analyste essaie de faire surgir. Un changement de position subjective a lieu pendant une analyse, ce que Lacan met en remarque en 1956 :

Je le disais schématiquement dans le temps archaïque de ces séminaires - le sujet commence par parler de lui, il ne parle pas à vous - ensuite, il parle à vous, mais il ne parle pas de lui - quand il aura parlé de lui, qui aura sensiblement changé dans l'intervalle, à vous, nous seront arrivés à la fin de l'analyse. (Lacan, 1955-1956/1981, p. 256) 
Celle-ci était la notion de fin d'analyse d'après Lacan dans la première phase de son enseignement. La perspective de sujet adoptée par Lacan à cette époque était toujours celle du sujet barré. Sur la position de l'analyste, encore sur le Séminaire III, il affirme qu'il s'agit de devoir être « assez mort pour ne pas être pris dans la relation imaginaire» (Lacan, 1955-1956/1981, p. 256).

Les conceptions de Lacan par rapport à la fin d'analyse ont changé au fur et à mesure de son enseignement, ainsi que la conception de sujet a aussi souffert des modifications. Le S barré était le résultat d'une perspective basée sur le symbolique, le Lacan de la chaîne signifiante. C'est-à-dire que cette conception de sujet a été formulée à travers ce que Lacan avait à l'époque comme source, à savoir, la structure du langage. Le problème est que cette perspective ne prend pas compte le corps de ce sujet, ne lui donne pas de consistance corporelle.

Dans un deuxième moment de l'enseignement, le corps et la jouissance prennent un poids différencié et Lacan fait un chemin vers la construction d'un sujet plutôt du côté du réel que du symbolique, de la jouissance et de lalangue. Le parlêtre intègre les dimensions du dit et du dire dans un seul concept, le sujet borroméen qu'à partir d'un trait de singularité fait tenir les trois registres RSI. Lacan passe d'une notion structurale et linguistique à une perspective plutôt mathématique et réel, au deuxième enseignement.

La jouissance, d'après Lacan, est « ce qui ne sert à rien »(Lacan, 1975, p. 10), mais qui ne cesse pas d'insister. Au sein du Séminaire XX, Lacan évoque que la substance du corps est la jouissance, et que l'expérience psychanalytique suppose proprement que la substance d'un corps se définit «seulement de ce qui se jouit » (Lacan, 1975, p. 26). Dans cette logique, il insert le signifiant comme substance jouissante et affirme que «le signifiant, c'est la cause de la jouissance. Sans le signifiant, comment même aborder cette partie du corps ? Comment, sans le signifiant, centrer ce quelque chose qui, de la jouissance, est la cause matérielle? »(Lacan, 1975, p. 27).

Le sinthome, de façon simple et résumée est justement le point d'attrapage de ce qu'il y a de plus singulier chez chaque sujet, et cette notion est fondamentale pour comprendre la fin d'analyse d'après Lacan aux années 1960. Compte tenu qu'aux années 1950 la fin d'analyse avait un rapport au signifiant, aux années 1960, Lacan propose qu'en fin d'analyse le sujet se confronte à l'objet, petit a. C'est objet qui a en même temps une fonction de reste de la division de sujet et de comblement est intégré dans la notion de fin d'analyse. Pas que ça, la notion de jouissance est aussi ajoutée dans le schéma signifiant, ce qui est éclairé avec Joyce (Lacan, 1975/2001a), celui qui peut jouir de la lettre.

«La cure analytique lacanienne est une expérience rigoureusement singulière mettant en jeu les inventions exceptionnelles d'un sujet» (Pernot, 2018, s.d., s.p.), 
et le deuxième enseignement focalise sur l'expérience du singulier de chaque sujet et la possibilité de bien-dire son symptôme, c'est-à-dire, mettre en place une nouvelle façon de parler de son histoire.

Le sujet du deuxième enseignement est borroméen et la topologie donne consistance à ce sujet qui fait tenir trois registres et qui manie ce nœud pendant un processus analytique. Le nœud borroméen est une " écriture qui supporte un réel » (Lacan, 1974, p. 100), et dans cette perspective, la fin d'analyse coïncide avec la possibilité d'écrire quelque chose de singulier face à l'impossibilité, au non-rapport sexuel.

Avec le Séminaire $X X$, Lacan met en cause la structure du langage, il évoque " un Autre mode du parlant dans le langage » (Lacan, 1975, p. 252), ce qui change tout, vu que le symptôme n'est plus considéré comme simple retour du refoulé, mais comme l'effet du symbolique dans le réel, le point où le corps se rejoint à la jouissance. C'est-à-dire, qu'à partir de cette époque-là, la fin d'analyse se trouve plutôt du côté d'une confrontation avec la jouissance et l'analyste est celui que fait un vidage de sens pour amener le sujet à atteindre le réel du symptôme, l'opacité de la jouissance.

Cette opération se déroule à travers l'équivoque comme source d'interprétation. L'équivoque et la position de l'analyste sont évoqués par Lacan en "Télévision ", quand il affirme que la position de l'analyste peut être comparé à un saint. Pas dans le sens où le saint fait de la charité, mais qu'il décharite. Je le cite : «Un saint, pour me faire comprendre, ne fait pas la charité. Plutôt se met-il à faire le déchet: il décharite. C'est pour réaliser ce que la structure impose, à savoir permettre au sujet, au sujet de l'inconscient, de le prendre pour cause de son désir » (Lacan, 1973/2001b, p. 519).

Dans le même texte, il affirme que « le sujet de l'inconscient, lui, embraye sur le corps » (Lacan, 2001b, p. 537) et cette notion nous montre que le parlêtre est une jonction de la jouissance et la parole, ce sujet ravagé par le verbe, qui fait du corps littoral. L'analyste, est celui qui fait un bon usage de la lettre et arrive à vider le sens et faire chuter le fantasme, ce qui fait que le sujet soit obligé à confronter le réel de sa jouissance. Quand le sujet ne s'en sert plus de son fantasme, il peut finalement avoir un autre rapport à son symptôme. Cette chute ouvre les portes à la possibilité de l'introduction du nouveau, de l'ordre de l'inventivité. Cette clinique invite les analystes à ne pas reculer devant le réel et à s'orienter par prendre le désir à la lettre. 


\section{Références bibliographiques}

Freud, S. (1978). Un enfant est battu : contribution à la connaissance de la genèse des perversions sexuelles. Paris : PUF. (Texte original publié en 1919)

Izcovich, L. (2008). L'être de jouissance. L’en-je Lacanien, 11, 2. Retrouvé en 2 septembre, 2018, de https://www.cairn.info/revue-l-en-je-lacanien-2008-2-page-35.htm\#

Lacan, J. (1958). Le séminaire VI : le désir et son interprétation. Paris : Seuil.

Lacan, J. (1966a). Le séminaire sur la lettre volée. In J. Lacan. Écrits. Paris : Seuil.

Lacan, J. (1966b). Subversion du sujet et dialectique du désir dans l'inconscient freudien. In J. Lacan. Écrits. Paris : Seuil.

Lacan, J. (1966c). L'étourdit. In J. Lacan. Autres écrits. Paris : Seuil.

Lacan, J. (1966d). La direction de la cure et les principles de son pouvoir. In J. Lacan. Écrits (pp. 585-646). Paris : Seuil. (Texte original publié en 1958)

Lacan, J. (1973). Le séminaire, livre XI : les quatre concepts fondamentaux de la psychanalyse. Paris : Seuil. (Texte original publié en 1964)

Lacan, J. (1974). Séminaire 22 : RSI. Séance du 17 décembre 1974. Ornicar ?, 2.

Lacan, J. (1975). Le séminaire XXI : encore. Paris : Seuil. (Texte original publié en 1972-1973)

Lacan, J. (1981). Le séminaire III : les psychoses, 1955-1956. Paris : Seuil. (Texte original publié en 1955-1956)

Lacan, J. (1991). Le séminaire XVII : l'envers de la psychanalyse. Paris : Seuil. (Texte original publié en 1969-1970)

Lacan, J. (1998). Le séminaire, livre V : les formations de l'inconscient. Paris : Seuil (Texte original publié en 1957-1958)

Lacan, J. (2001a). Joyce le symptôme. In J. Lacan. Autres écrits. Paris : Seuil. (Texte original publié en 1975)

Lacan, J. (2001b). Télévision. In J. Lacan. Autres écrits. Paris : Seuil. (Texte original publié en 1973)

Lacan, J. (2001c). Radiophonie. In J. Lacan. Autres écrits. Paris : Seuil. (Texte original publié en 1970)

Lacan, J. (2001d). L'étourdit. In J. Lacan. Autres écrits. Paris : Seuil. (Texte original publié en 1972)

Lacan, J. (2013). Le séminaire VI : désir et son interprétation. Paris : Editions de Martinière. (Texte original publié en 1958-1959) 
Miller, J.-A. (1983). Des réponses du réel. Cours du 16 novembre 1983. Paris : Seuil. Pernot, P. Du sujet de l'inconscient au parlêtre. Cartel de Monique Amirault, Serge Cottet, Claude Quénardel, Marie-Hélène Roch, Pascal Pernot. In Lectures théoriques. [s.d.]. Ecole de la Cause Freudienne. Retrouvé en 20 novembre, 2018, de https://www.causefreudienne.net/du-sujet-de-linconscient-au-parletre/

Recebido: 06/03/2019

Aprovado: 15/05/2019 